TRANSACTIONS OF THE

AMERICAN MATHEMATICAL SOCIETY

Volume 352, Number 12, Pages 5563-5582

S 0002-9947(00)02502-2

Article electronically published on August 21, 2000

\title{
ASYMPTOTIC RELATIONS AMONG FOURIER COEFFICIENTS OF REAL-ANALYTIC EISENSTEIN SERIES
}

\author{
ALVARO ALVAREZ-PARRILLA
}

\begin{abstract}
Following Wolpert, we find a set of asymptotic relations among the Fourier coefficients of real-analytic Eisenstein series. The relations are found by evaluating the integral of the product of an Eisenstein series $\varphi_{i r}$ with an exponential factor along a horocycle. We evaluate the integral in two ways by exploiting the automorphicity of $\varphi_{i r}$; the first of these evaluations immediately gives us one coefficient, while the other evaluation provides us with a sum of Fourier coefficients. The second evaluation of the integral is done using stationary phase asymptotics in the parameter $\lambda\left(\lambda=\frac{1}{4}+r^{2}\right.$ is the eigenvalue of $\varphi_{i r}$ ) for a cubic phase.
\end{abstract}

As applications we find sets of asymptotic relations for divisor functions.

\section{Preliminaries}

Let $\Gamma \subset S L_{2}(\mathbb{R})$ be a finitely generated non-cocompact Fuchsian group of the first kind 1 For ease of presentation, we shall restrict ourselves to the case where $\Gamma=S L_{2}(\mathbb{Z})$, although most of the work carries through to the general case (the exception being in the Appendix, where one has to be careful about the choice of group so that it has a standard fundamental domain).

Let $\varphi_{s-\frac{1}{2}}$ denote a $\Gamma$-automorphic eigenfunction of the hyperbolic Laplacian with eigenvalue $\lambda=s(1-s)=\frac{1}{4}+r^{2}>\frac{1}{4}$ on the critical line $\operatorname{Re}(s)=\frac{1}{2}$ (we choose $s$ such that $\left.s=\frac{1}{2}+i r\right)$ with a Fourier series development of the form

$$
\varphi_{s-\frac{1}{2}}(z)=a_{0_{+}} y^{s}+a_{0_{-}} y^{1-s}+\sum_{m \neq 0} a_{m} y^{\frac{1}{2}} K_{s-\frac{1}{2}}(2 \pi|m| y) \mathrm{e}^{i 2 \pi m x},
$$

where $K_{q}$ denotes the $K$-Bessel functions (also known as the MacDonald-Bessel functions), and $z=x+i y \in \mathbb{H}=\{z \in \mathbb{C}: y=\operatorname{Im}(z)>0\}$.

By saying that $\varphi_{i r}$ is $\Gamma$-automorphic for $\Gamma \subset S L_{2}(\mathbb{R})$, we mean that for $\gamma=$ $\left(\begin{array}{ll}a & b \\ c & d\end{array}\right) \in \Gamma \subset S L_{2}(\mathbb{R})$, acting on the upper half plane $\mathbb{H}$ by linear fractional transformations $z \mapsto \frac{a z+b}{c z+d}$, we have $\varphi_{i r}(z)=\varphi_{i r}(\gamma z)$. In the case that $\Gamma=S L_{2}(\mathbb{Z})$, we

Received by the editors September 29, 1998 and, in revised form, November 24, 1998 and January 29, 1999.

1991 Mathematics Subject Classification. Primary 11F30; Secondary 11N37.

Key words and phrases. Automorphic forms, Eisenstein series, microlocal analysis, divisor functions.

Thanks to Scott Wolpert for suggesting the problem, many very insightful talks and helpful ideas, and for providing copies of his preprint.

${ }^{1}$ In other words $\Gamma$ is a subgroup of $P S L_{2}(\mathbb{R})$ acting discontinuously on $\mathbb{H}$ containing parabolic elements, and every point on the boundary $\partial \mathbb{H}$ is a limit of an orbit $\Gamma z$ for some $z \in \mathbb{H}$. Poincaré showed that a subgroup of $S L_{2}(\mathbb{R})$ is discrete if and only if it acts discontinuously (when considered as a subgroup of $\left.P S L_{2}(\mathbb{R})\right)$. 
have in mind that $\varphi_{i r}(z)$ be the classical Eisenstein series

$$
\varphi_{s-\frac{1}{2}}(z)=\frac{1}{2} E(z, s)=\frac{1}{2} \sum_{\substack{c, d \in \mathbb{Z} \\(c, d)=1}} y^{s}|c z+d|^{-2 s} .
$$

Let $t=2 \pi n \lambda^{-\frac{1}{2}}$, for $n>0$, and consider the integral over the horocycle $h o r_{\lambda}=$ $\{z \in \mathbb{H}: t y=1\}$,

$$
\mathcal{J}=\int_{t y=1} \varphi_{i r}(z) \mathrm{e}^{2 \pi i n x} h(x) d x,
$$

where $h$ is a smooth function with compact support (say $\operatorname{Support}(h) \subset\left(h_{0}, h_{1}\right)$ with $\left.h_{1}-h_{0}<2\right)$ and satisfying

$$
\sum_{k \in \mathbb{Z}} h(x+k) \equiv 1 .
$$

From (1.1), and using the fact that $h$ has compact support, we get

$$
\begin{aligned}
\mathcal{J}= & \int_{t y=1} \varphi_{i r}(z) \mathrm{e}^{i 2 \pi n x} h(x) d x \\
= & \int_{t y=1} a_{0}(y) \mathrm{e}^{i 2 \pi n x} h(x) d x \\
& +\sum_{m \neq 0} \int_{t y=1} a_{m}(y) \mathrm{e}^{i 2 \pi(m+n) x} h(x) d x \\
= & \mathcal{I}_{0}+\sum_{m \neq 0} \mathcal{I}_{m},
\end{aligned}
$$

where

$$
a_{0}(y)=a_{0_{+}} y^{\frac{1}{2}+i r}+a_{0 \_} y^{\frac{1}{2}-i r}
$$

and for $m \neq 0$

$$
a_{m}(y)=a_{m} y^{1 / 2} K_{i r}(2 \pi|m| y) .
$$

On the other hand, let $\gamma \in \Gamma$, and let

$$
\mathcal{J}^{\prime}=\int_{t y=1} \varphi_{i r}(\gamma z) \mathrm{e}^{i 2 \pi n x} h(x) d x .
$$

Hence we have, in a similar manner as before,

$$
\begin{aligned}
\mathcal{J}^{\prime}= & \int_{t y=1} \varphi_{i r}(\gamma z) \mathrm{e}^{i 2 \pi n x} h(x) d x \\
= & \int_{t y=1} a_{0}(\operatorname{Im}(\gamma z)) \mathrm{e}^{i 2 \pi n x} h(x) d x \\
& +\sum_{m \neq 0} \int_{t y=1} a_{m}(\operatorname{Im}(\gamma z)) \mathrm{e}^{i 2 \pi \operatorname{Re}(m \gamma z+n z)} h(x) d x \\
= & \mathcal{I}_{0}^{\prime}+\sum_{m \neq 0} \mathcal{I}_{m}^{\prime} .
\end{aligned}
$$


Since $\varphi_{\text {ir }}$ is automorphic it follows that $\mathcal{J}=\mathcal{J}^{\prime}$, and the coefficient relation (Corollary [5.2)

$$
\begin{aligned}
a_{n} \mathrm{e}^{-\pi \lambda^{\frac{1}{2}} / 2}= & 2^{\frac{2}{3}} t^{\frac{1}{2}} \mathcal{A}(c, d) \mathrm{e}^{-i \lambda^{\frac{1}{2}} Q_{0}(\rho)} g(0) \\
+ & +2^{\frac{1}{6}} 3^{-\frac{1}{3}} \pi^{\frac{1}{2}} \lambda^{-\frac{1}{4}} \sum_{m>0} a_{m} \mathrm{e}^{-\pi \lambda^{\frac{1}{2}} / 2} s^{-\frac{1}{2}} t^{\frac{1}{2}} \mathrm{e}^{-i \lambda^{\frac{1}{2}} \widehat{Q_{0}}+i \frac{\pi}{4}} \\
& \quad \times\left(\overline{G_{0}(\tau, t)}-i \frac{\Gamma\left(\frac{2}{3}\right)}{\Gamma\left(\frac{1}{3}\right)} \frac{\overline{G_{1}(\tau, t)}}{\lambda^{1 / 6}}\right)+O\left(\lambda^{-1 / 12}\left\|\varphi_{i r}^{Y}\right\|_{L_{2}}\|h\|_{H^{4}}\right)
\end{aligned}
$$

is obtained by equating the evaluation of the integrals $\mathcal{J}$, and $\mathcal{J}^{\prime}$.

The considerations are organized as follows: In $\$ 2$ we obtain the stationary phase expansion for the integral of an exponential with a general cubic phase, in $\$ 3$ we evaluate the integrals comprising $\mathcal{J}$, and in $\$$ we evaluate the integrals comprising $\mathcal{J}^{\prime}$. It is relevant to notice that the evaluation of $\mathcal{J}^{\prime}$ involves a stationary phase analysis uniformly valid on a noncompact set of parameter values. In \$5 we join the two evaluations to obtain the final coefficient relation.

In 6 we explore some applications of the coefficient relation. In particular, if $d_{q}(n)=\sum_{d \mid n} d^{q}$ denotes the divisor function, then, using the classical Eisenstein series (1.2), we obtain the following asymptotic recursion relation (Corollary 6.2):

$$
\begin{aligned}
& \frac{\mathrm{d}_{2 i r}(n)}{n^{\frac{1}{2}+i r}}=2^{\frac{1}{6}} \pi^{-i r} \lambda^{-\frac{1}{4}} \mathcal{B}(c, d) \mathrm{e}^{-i \lambda^{\frac{1}{2}} Q_{0}(\rho)} g(0) \\
& +2^{\frac{1}{6}} 3^{-\frac{1}{3}} \pi^{\frac{1}{2}} \lambda^{-\frac{1}{4}} \sum_{m>0} \frac{\mathrm{d}_{2 i r}(m)}{m^{\frac{1}{2}+i r}} \mathrm{e}^{-i \lambda^{\frac{1}{2}} \widehat{Q_{0}}+i \frac{\pi}{4}}\left(\overline{G_{0}(\tau, t)}+i \frac{\Gamma\left(\frac{2}{3}\right)}{\Gamma\left(\frac{1}{3}\right)} \frac{\overline{G_{1}(\tau, t)}}{\lambda^{1 / 6}}\right) \\
& +O\left(\lambda^{-1 / 12}\left\|\varphi_{i r}^{Y}\right\|_{L_{2}}\|h\|_{H^{4}}\right) .
\end{aligned}
$$

\section{Stationary Phase}

In this section we obtain the stationary phase asymptotic expansion for the integral of an exponential with a cubic phase function that has two coalescing stationary points.

2.1. Setup. We consider integrals of the type

$$
\int_{-\infty}^{\infty} g(x) \mathrm{e}^{i \nu\left(\frac{x^{3}}{3}-\alpha x\right)} d x,
$$

where $g(x)$ is a real valued $C^{\infty}$ function with compact support containing a neighborhood of the origin, and $\alpha=\alpha(\rho)$ is an analytic function of the parameter $\rho \in \mathbb{C}$ such that $\alpha \rightarrow 0$ as $\rho \rightarrow \rho_{0}$.

We are interested in obtaining the asymptotic expansion of this integral for large $\nu$, uniformly in a domain containing $\rho_{0}$. Notice that the regularity of $\alpha$ guarantees this last statement. The stationary points of the phase for this integral are $\pm \sqrt{\alpha}$. As $\rho \rightarrow \rho_{0}, \alpha \rightarrow 0$; hence the stationary points coalesce into one stationary point.

2.2. Results. Let $g$ be a function on $\mathbb{R}$, and define the Fourier transform of $g$ by $\mathcal{F}(g)(t)=\widehat{g}(t)=\int_{-\infty}^{\infty} g(x) \mathrm{e}^{-i x t} d x$ whenever the integral converges. Let $\mathcal{S}$ denote Schwartz's functions, rapidly decaying smooth functions; then $\mathcal{F}: \mathcal{S} \rightarrow \mathcal{S}$ is an isometry. For a tempered distribution $T$ we define the Fourier distribution of $T$ by

$$
\langle\mathcal{F}(T), \varphi\rangle=\langle T, \mathcal{F}(\varphi)\rangle, \quad \forall \varphi \in \mathcal{S}
$$


where $\langle f, g\rangle=\int_{-\infty}^{\infty} f(x) \overline{g(x)} d x$ (see [Zem65] for a discussion of tempered distributions and Fourier transforms).

The following results follow directly from the definition of the Fourier transform and its properties, as well as from the definition of the Airy function (for a discussion of the Airy function see for instance [Olv97]).

Lemma 2.1.

$$
\mathcal{F}\left(\mathrm{e}^{i \nu\left(\frac{x^{3}}{3}-\alpha x\right)}\right)(t)=2 \pi \nu^{-\frac{1}{3}} A i\left(-\alpha \nu^{2 / 3}-\nu^{-1 / 3} t\right) .
$$

Proof. We have

$$
\begin{aligned}
\mathcal{F}\left(\mathrm{e}^{i \nu\left(\frac{x^{3}}{3}-\alpha x\right)}\right)(t) & =\int_{-\infty}^{\infty} \mathrm{e}^{i\left(\frac{\nu x^{3}}{3}-(\alpha \nu+t) x\right)} d x \\
& =\nu^{-1 / 3} \int_{-\infty}^{\infty} \mathrm{e}^{i\left(\frac{\tau^{3}}{3}-\left(\alpha \nu^{2 / 3}+\nu^{-1 / 3} t\right) \tau\right)} d \tau \\
& =2 \pi \nu^{-\frac{1}{3}} A i\left(-\alpha \nu^{2 / 3}-\nu^{-1 / 3} t\right)
\end{aligned}
$$

since by definition

$$
2 \pi A i(\mu)=\int_{-\infty}^{\infty} \mathrm{e}^{i\left(\frac{\tau^{3}}{3}+\mu \tau\right)} d \tau
$$

Proposition 2.2. Let $\nu>0, g \in C_{c}^{\infty}, \alpha=O\left(\nu^{-1-\epsilon}\right)$ for some $\epsilon>0$, and $N a$ non-negative integer. Then

$$
\begin{aligned}
\mathcal{I}= & \int_{-\infty}^{\infty} g(x) \mathrm{e}^{i \nu\left(\frac{x^{3}}{3}-\alpha x\right)} d x \\
= & 2 \pi \nu^{-1 / 3} \sum_{n=0}^{N} \frac{i^{n} A i^{(n)}(0)}{\nu^{n / 3} n !} \sum_{j=0}^{n}\left(\begin{array}{c}
n \\
j
\end{array}\right)(i \alpha \nu)^{n-j} g^{(j)}(0) \\
& \quad+O_{N}\left(\nu^{-(N+2) / 3} \sum_{j=0}^{N+1}\left(\begin{array}{c}
N+1 \\
j
\end{array}\right)|\alpha \nu|^{N+1-j}\left(\|g\|_{L_{1}}+\left\|g^{(j+2)}\right\|_{L_{1}}\right)\right)
\end{aligned}
$$

is an asymptotic expansion for $\nu$ large.

Proof. First apply Parseval's identity to $\mathcal{I}$, to obtain

$$
\begin{aligned}
\mathcal{I} & =\int_{-\infty}^{\infty} g(x) \mathrm{e}^{i \nu\left(\frac{x^{3}}{3}-\alpha x\right)} d x \\
& =\frac{1}{2 \pi} \int_{-\infty}^{\infty} \widehat{g}(t) \mathcal{F}\left(\mathrm{e}^{i \nu\left(\frac{x^{3}}{3}-\alpha x\right)}\right)(t) d t \\
& =\nu^{-1 / 3} \int_{-\infty}^{\infty} \widehat{g}(t) A i\left(-\alpha \nu^{2 / 3}-\nu^{-1 / 3} t\right) d t
\end{aligned}
$$

the last equality stemming from Lemma 2.1

Let $\tau=-\alpha \nu^{2 / 3}-\nu^{-1 / 3} t$; then

$$
\mathcal{I}=\int_{-\infty}^{\infty} \widehat{g}\left(-\alpha \nu-\nu^{1 / 3} \tau\right) A i(\tau) d \tau
$$

Then from Taylor's theorem we have

$$
\left|A i(\tau)-P_{N}(\tau)\right| \leq C_{N}|\tau|^{N+1}, \quad \forall \tau,
$$


where $P_{N}(\tau)=\sum_{n=0}^{N} \frac{A i^{(n)}(0)}{n !} \tau^{n}$ is the $N$-th Taylor polynomial of $A i$. Thus

$$
\begin{aligned}
\mathcal{I}=\sum_{n=0}^{N} \frac{A i^{(n)}(0)}{n !} \int_{-\infty}^{\infty} \widehat{g}\left(-\alpha \nu-\nu^{1 / 3} \tau\right) \tau^{n} d \tau \\
+O_{N}\left(\int_{-\infty}^{\infty}\left|\widehat{g}\left(-\alpha \nu-\nu^{1 / 3} \tau\right) \tau^{N+1}\right| d \tau\right) .
\end{aligned}
$$

On the other hand,

$$
\begin{aligned}
\int_{-\infty}^{\infty} \widehat{g}\left(-\alpha \nu-\nu^{1 / 3} \tau\right) \tau^{n} d \tau & =\nu^{-1 / 3} \int_{-\infty}^{\infty} \widehat{g}(t)\left(-\alpha \nu^{2 / 3}-\nu^{-1 / 3} t\right)^{n} d t \\
& =2 \pi i^{n} \nu^{-(n+1) / 3} \sum_{j=0}^{n}\left(\begin{array}{c}
n \\
j
\end{array}\right)(i \alpha \nu)^{n-j} g^{(j)}(0)
\end{aligned}
$$

where we have used the binomial theorem and the following property of the Fourier transform:

$$
(i t)^{k} \widehat{g}(t)=\widehat{g^{(k)}}(t)=\int_{-\infty}^{\infty} g^{(k)}(x) \mathrm{e}^{-i x t} d x
$$

so that $\int_{-\infty}^{\infty}(i t)^{j} \widehat{g}(t) d t=2 \pi g^{(j)}(0)$.

Similarly we have that

$$
\begin{gathered}
\int_{-\infty}^{\infty}\left|\widehat{g}\left(-\alpha \nu-\nu^{1 / 3} \tau\right) \tau^{N+1}\right| d \tau=\nu^{-1 / 3} \int_{-\infty}^{\infty}\left|\widehat{g}(t)\left(-\alpha \nu^{2 / 3}-\nu^{-1 / 3} t\right)^{N+1}\right| d t \\
\leq \nu^{-(N+2) / 3} \sum_{j=0}^{N+1}\left(\begin{array}{c}
N+1 \\
j
\end{array}\right)|\alpha \nu|^{N+1-j} \int_{-\infty}^{\infty}\left|\widehat{g}(t)(i t)^{j}\right| d t
\end{gathered}
$$

Once again, using (2.10) we have that, for $k$ an integer, $\left|t^{k} \widehat{g}(t)\right| \leq\left\|g^{(k)}\right\|_{L_{1}}$. Thus, in particular, for $|t| \geq 1$

$$
\left|t^{j} \widehat{g}(t)\right| \leq\left\|g^{(j)}\right\|_{L_{1}} \quad \forall j \geq 0 .
$$

On the other hand, for $|t| \leq 1$

$$
\left|t^{j} \widehat{g}(t)\right| \leq|\widehat{g}(t)| \leq \int_{-\infty}^{\infty}\left|g(x) \mathrm{e}^{-i x t}\right| d x=\|g\|_{L_{1}} .
$$

Hence

$$
\left|t^{j} \widehat{g}(t)\right| \leq \begin{cases}\frac{\left\|g^{(j+2)}\right\| \|_{L_{1}}}{|t|^{2}}, & |t| \geq 1 \\ \|g \mid\|_{L_{1}}, & |t| \leq 1\end{cases}
$$

Thus

$$
\begin{aligned}
\int_{-\infty}^{\infty}\left|\widehat{g}(t)(i t)^{j}\right| d t & =\int_{-1}^{1}\|g\|_{L_{1}} d t+2 \int_{1}^{\infty} \frac{\left\|g^{(j+2)}\right\|_{L_{1}}}{t^{2}} d t \\
& \leq 2\left(\|g\|_{L_{1}}+\left\|g^{(j+2)}\right\|_{L_{1}}\right),
\end{aligned}
$$


so substituting this result in (2.11) we obtain

$$
\begin{aligned}
\int_{-\infty}^{\infty} \mid \widehat{g}(-\alpha \nu & \left.-\nu^{1 / 3} \tau\right) \tau^{N+1} \mid d \tau \\
& \leq 2 \nu^{-(N+2) / 3} \sum_{j=0}^{N+1}\left(\begin{array}{c}
N+1 \\
j
\end{array}\right)|\alpha \nu|^{N+1-j}\left(\|g\|_{L_{1}}+\left\|g^{(j+2)}\right\|_{L_{1}}\right) .
\end{aligned}
$$

Finally, substitution of (2.9) and (2.16) in (2.8) finishes the proof.

Remark 2.3. In the proposition above it is understood that the quantity $0^{0}$ is 1 by continuity.

Notice that when $\alpha=0$ we recover Proposition 2.6 of Wolpert Wol99.

\section{Evaluation of $\mathcal{J}$}

In this section we evaluate the integral $\mathcal{J}$. As can be seen from (1.5) and from (1.6) and (1.7), we need to evaluate two types of integrals: those corresponding to the the zeroth order coefficient and those corresponding to the non-zero order coefficients of the Fourier development of $\varphi_{i r}$. The argument used to evaluate them is basically the same.

The summation condition $\sum_{k \in \mathbb{Z}} h(x+k) \equiv 1$ for the test function $h$ plays an essential role in this computation.

3.1. Zeroth order coefficient. We start by considering

$$
\begin{aligned}
\mathcal{I}_{0} & =\int_{t y=1}\left(a_{0_{+}} y^{1 / 2+i r}+a_{0_{-}} y^{1 / 2-i r}\right) \mathrm{e}^{i 2 \pi n x} h(x) d x \\
& =a_{0_{+}} \mathcal{I}_{+}+a_{0_{-}} \mathcal{I}_{-},
\end{aligned}
$$

where for $\delta= \pm 1$ we have the integrals

$$
\mathcal{I}_{\delta}=\int_{t y=1} y^{\frac{1}{2}+\delta i r} \mathrm{e}^{i 2 \pi n x} h(x) d x .
$$

Proposition 3.1. $\mathcal{I}_{0}=0$.

Proof. From (3.1) we need only evaluate $\mathcal{I}_{\delta}$ :

$$
\begin{aligned}
\mathcal{I}_{\delta} & =\int_{t y=1} y^{\frac{1}{2}+\delta i r} \mathrm{e}^{i 2 \pi n x} h(x) d x \\
& =\int_{-\infty}^{\infty} t^{-\left(\frac{1}{2}+\delta i r\right)} \mathrm{e}^{i 2 \pi n x} h(x) d x \\
& =\sum_{k \in \mathbb{Z}} \int_{k}^{k+1} t^{-\left(\frac{1}{2}+\delta i r\right)} \mathrm{e}^{i 2 \pi n x} h(x) d x \\
& =t^{-\left(\frac{1}{2}+\delta i r\right)} \int_{0}^{1} \mathrm{e}^{i 2 \pi n x} \sum_{k \in \mathbb{Z}} h(x+k) d x \\
& =t^{-\left(\frac{1}{2}+\delta i r\right)} \int_{0}^{1} \mathrm{e}^{i 2 \pi n x} d x,
\end{aligned}
$$

where we have used that $h$ has compact support, $\mathrm{e}^{i 2 \pi n x}$ is translation invariant, and $\sum_{k \in \mathbb{Z}} h(x+k) \equiv 1$. The statement now follows from the fact $n \neq 0$. 
3.2. Higher order coefficients. The evaluation of the integrals

$$
\sum_{m \neq 0} \mathcal{I}_{m}=\sum_{m \neq 0} \int_{t y=1} a_{m} y^{\frac{1}{2}} K_{i r}(2 \pi|m| y) \mathrm{e}^{i 2 \pi(m+n) x} h(x) d x
$$

has been done by Wolpert in Wol99 and can be found in the proof of Corollary 4.9. Here we state the result and provide a sketch of the proof.

Remark 3.2. The notation is as in Wol99. The proof rests on Lemma 2.3 of Wol99, which, in a nutshell, provides an asymptotic expansion for the product $\sqrt{2 \pi|m| y} K_{i r}(2 \pi|m| y)$ in terms of the Airy function and its derivative.

Proposition 3.3 (Wolpert). For $\lambda$ large and $n>0$,

$$
\sum_{m \neq 0} \mathcal{I}_{m}=\overline{a_{n}} 2^{\frac{1}{3}} \pi t^{-1 / 2} \lambda^{-1 / 6} \mathrm{e}^{-\pi \lambda^{1 / 2} / 2}\left(A i(0)+\frac{\lambda^{-2 / 3} 2^{1 / 3} A i^{\prime}(0)}{70}+O\left(\lambda^{-1}\right)\right) .
$$

Proof. As in Proposition 3.1, $\mathrm{e}^{i 2 \pi(m+n) x}$ is unit-translation invariant, so the limits of integration can be replaced by $\{0,1\}$ provided that we also replace $h(w)$ by $\sum_{k \in \mathbb{Z}} h(w+k) \equiv 1$. Then orthogonality of exponentials, an application of Lemma 2.3 of Wol99, and some further estimates finish the proof.

3.3. Putting together Proposition 3.3 and Proposition 3.1, we obtain

Corollary 3.4. For $\lambda$ large and $n>0$,

$$
\mathcal{J}=\overline{a_{n}} 2^{\frac{1}{3}} \pi t^{-1 / 2} \lambda^{-1 / 6} \mathrm{e}^{-\pi \lambda^{1 / 2} / 2}\left(A i(0)+\frac{\lambda^{-2 / 3} 2^{1 / 3} A i^{\prime}(0)}{70}+O\left(\lambda^{-1}\right)\right) .
$$

\section{Evaluation of $\mathcal{J}^{\prime}$}

In this section we evaluate the integral $\mathcal{J}^{\prime}$. From (1.9), (1.6) and (1.7), we again see the need to evaluate two different types of integrals: those corresponding to the the zeroth order coefficient and those corresponding to the non-zero order coefficients of the Fourier development of $\varphi_{i r}$.

Notice that we do not use the summation condition for the test function $h$.

4.1. Zeroth order coefficient. By (1.9) and (1.6) we have

$$
\begin{aligned}
\mathcal{I}_{0}^{\prime} & =\int_{t y=1} a_{0}(\operatorname{Im}(\gamma z)) \mathrm{e}^{i 2 \pi n x} h(x) d x \\
& =a_{0_{+}} \mathcal{I}_{+}^{\prime}+a_{0_{-}} \mathcal{I}_{-}^{\prime},
\end{aligned}
$$

where for $\delta= \pm 1$ we have the integrals

$$
\begin{aligned}
\mathcal{I}_{\delta}^{\prime} & =\int_{t y=1}(\operatorname{Im}(\gamma z))^{\frac{1}{2}+\delta i r} \mathrm{e}^{i 2 \pi n x} h(x) d x \\
& =\int_{t y=1}\left(\frac{y}{|c z+d|}\right)^{\frac{1}{2}} \mathrm{e}^{i \lambda^{\frac{1}{2}} \psi_{\delta}} h(x) d x,
\end{aligned}
$$

where the 'phase function' $\psi_{\delta}$ is defined by

$$
\psi_{\delta}=\frac{\delta}{\rho} \log \left(\frac{y}{|c z+d|}\right)+t x,
$$

where

$$
\rho=\frac{\lambda^{\frac{1}{2}}}{r}=\sqrt{1+\frac{1}{4 r^{2}}} \geq \rho_{0}=1
$$


( $\rho_{0}=1$ corresponds to the limit $\left.\lambda \rightarrow \infty\right)$, and we recall that $t=2 \pi n \lambda^{-\frac{1}{2}}>0$, since $n>0$.

To evaluate the integrals $\mathcal{I}_{\delta}^{\prime}$ we will use stationary phase analysis. Thus we first make a detailed analysis of the phase function $\psi_{\delta}$ on the horocycle hor $_{\lambda}$.

4.1.1. Analysis of the phase function $\psi_{\delta}$. On the horocycle hor ${ }_{\lambda}$ we have $t y=1$. Hence

$$
\psi_{\delta}(x, \rho)=\delta \rho^{-1} \log \left(\frac{t}{(c x+d)^{2} t^{2}+c^{2}}\right)+t x
$$

Let

$$
\sigma=\frac{\delta t}{c}(c x+d)
$$

then $\frac{d \sigma}{d x}=\delta t$, and

$$
\psi_{\delta}=-t \frac{d}{c}+\delta\left(\sigma+\rho^{-1} \log \left(\frac{t}{c^{2}\left(\sigma^{2}+1\right)}\right)\right) .
$$

Furthermore, let

$$
\widetilde{Q_{0}}=\left.\psi_{\delta}\right|_{\sigma=1}=-t \frac{d}{c}+\delta\left(1+\rho^{-1} \log \left(\frac{t}{2 c^{2}}\right)\right),
$$

and

$$
\phi(\sigma)=\delta\left(\sigma-1+\rho^{-1} \log \left(\frac{2}{\sigma^{2}+1}\right)\right) .
$$

Then $\psi_{\delta}=\phi+\widetilde{Q_{0}}$, where we note that $\widetilde{Q_{0}}$ is independent of $\sigma$. Note that $\phi$ vanishes at $\sigma=1$, and if $\phi^{(k)}(\sigma)=\frac{\partial^{k}}{\partial \sigma^{k}} \phi(\sigma)$ denotes the $k$-th derivative with respect to $\sigma$, then

$$
\begin{aligned}
\phi^{(1)}(\sigma) & =\delta \frac{\sigma^{2}-2 \rho^{-1} \sigma+1}{\sigma^{2}+1}, \\
\phi^{(2)}(\sigma) & =\frac{2 \delta}{\rho} \frac{(\sigma-1)(\sigma+1)}{\left(\sigma^{2}+1\right)^{2}}, \\
\phi^{(3)}(\sigma) & =-\frac{4 \delta}{\rho} \frac{\sigma\left(\sigma^{2}-3\right)}{\left(\sigma^{2}+1\right)^{3}} .
\end{aligned}
$$

Abusing notation, we denote the analytic continuation of $\phi$ by $\phi$. Its domain is given by $\mathcal{D}=\left\{(\sigma, \rho) \in \mathbb{C}^{2}: \sigma \neq i \nu, \nu \geq 1, \rho \neq 0\right\}$. Furthermore, as can be seen from (4.9), the critical points of $\phi(\sigma)$ are given by $\sigma_{ \pm}=\frac{1 \pm i \sqrt{\rho^{2}-1}}{\rho}$. It follows that $\phi$ has two critical points $\sigma_{ \pm}$for $\rho>\rho_{0}=1$, which coalesce to an order 3 zero of $\phi$ at $\sigma_{0}=1$ (when $\rho=\rho_{0}=1$ ).

Proposition 4.1. The phase function $\psi_{\delta}$ can be rewritten as

$$
\psi_{\delta}(x, \rho)=\frac{u^{3}}{3}-\alpha(\rho) u+Q_{0}(\rho),
$$

where $\alpha(\rho)$ and $Q_{0}(\rho)$ are constants depending on the parameter $\rho$, and $u=u(x, \rho)$ is an analytic function of $x$ and $\rho$, invertible with respect to $x$ for $\rho$ near $\rho_{0}$ and 
small $x$. Furthermore, $\alpha(\rho) \rightarrow 0$ and $Q_{0}(\rho) \rightarrow \widetilde{Q_{0}}$ when $\rho \rightarrow 1$. Explicitly, we have

$$
\begin{aligned}
\alpha(\rho) & =-\left(\frac{3}{2}\right)^{2 / 3}\left(\frac{\sqrt{\rho^{2}-1}-\arctan \sqrt{\rho^{2}-1}}{\rho}\right)^{2 / 3}, \\
Q_{0}(\rho) & =\frac{\delta}{\rho}\left(1+\log \left(\frac{t \rho^{2}}{2 c^{2}}\right)\right)-t \frac{d}{c} .
\end{aligned}
$$

Proof. From (4.5), clearly $\sigma=\sigma(x)$ is an invertible and analytic function of $x$; hence $\psi_{\delta}$ has the same behavior as $\phi$. So it is enough to consider $\phi$ and $\sigma$ (keeping in mind that we have to add the extra constant $\widetilde{Q_{0}}$ since $\psi_{\delta}=\phi+\widetilde{Q_{0}}$ ), and show that the transformation

$$
\mathcal{T}_{\rho}: \phi(\sigma, \rho)=\frac{u^{3}}{3}-\alpha(\rho) u+Q_{0}^{\prime}(\rho)
$$

where $Q_{0}=Q_{0}^{\prime}+\widetilde{Q_{0}}$, has a branch which is uniformly regular for $\rho$ and $\sigma$ in small neighborhoods of $\rho_{0}=1$ and $\sigma_{0}=1$, and that, in this same branch, for $\rho$ near enough to $\rho_{0}$ the implicit correspondence $u \leftrightarrow \sigma$ is $1-1$.

The proof of this last statement for $\phi$ satisfying (4.9) is well known and can be found as Theorem 1 in [CFU57 and in more generality for smooth functions in GS77. We find the functions $\alpha(\rho)$ and $Q_{0}(\rho)$ explicitly for our case.

In order for (4.10) to be a uniformly regular 1-1 transformation we must have that $\frac{d \sigma}{d u} \neq 0, \infty$, where

$$
\phi^{(1)}(\sigma, \rho) \frac{d \sigma}{d u}=u^{2}-\alpha(\rho) .
$$

Notice that the LHS vanishes at $\sigma_{ \pm}$, while the RHS has its zeros at $\pm \sqrt{\alpha}$. If the transformation is to be regular, these points must correspond, so by (4.10) we have

$$
\begin{aligned}
& \phi\left(\sigma_{+}, \rho\right)=-\frac{2}{3} \alpha(\rho)^{\frac{3}{2}}+Q_{0}^{\prime}(\rho), \\
& \phi\left(\sigma_{-}, \rho\right)=\frac{2}{3} \alpha(\rho)^{\frac{3}{2}}+Q_{0}^{\prime}(\rho) .
\end{aligned}
$$

Hence

$$
\alpha=\left(\frac{3}{2}\right)^{2 / 3}\left(\frac{\phi\left(\sigma_{-}\right)-\phi\left(\sigma_{+}\right)}{2}\right)^{2 / 3}
$$

and

$$
Q_{0}^{\prime}=\frac{\phi\left(\sigma_{-}\right)+\phi\left(\sigma_{+}\right)}{2} .
$$

Upon substitution of $\sigma_{ \pm}$in (4.8) and simplification of 4.13) and (4.14) we obtain explicit expressions for $\alpha$ and $Q_{0}^{\prime}$, namely

$$
\alpha(\rho)=-\left(\frac{3}{2}\right)^{2 / 3}\left(\frac{\sqrt{\rho^{2}-1}-\arctan \sqrt{\rho^{2}-1}}{\rho}\right)^{2 / 3},
$$

and

$$
Q_{0}^{\prime}(\rho)=\frac{\delta}{\rho}\left(1-\rho+\log \rho^{2}\right)
$$


Finally, since $Q_{0}=Q_{0}^{\prime}+\widetilde{Q_{0}}$, then

$$
Q_{0}(\rho)=\frac{\delta}{\rho}\left(1+\log \left(\frac{t \rho^{2}}{2 c^{2}}\right)\right)-t \frac{d}{c} .
$$

The last statement of the proposition follows from the explicit formulas for $\alpha(\rho)$ and $Q_{0}(\rho)$ (or equivalently from (4.14) and noting that $\phi(1)=0$ ).

Remark 4.2. Since $\rho=\sqrt{\frac{\lambda}{\lambda-\frac{1}{4}}}$, from (4.15) we see that, for $\lambda$ large,

$$
\alpha=O\left(\lambda^{-1}\right) .
$$

4.1.2. Evaluation of $\mathcal{I}_{\delta}^{\prime}$. We now proceed to evaluate the integral $\mathcal{I}_{\delta}^{\prime}$.

By Proposition 4.1 and using (4.5), the integral $\mathcal{I}_{\delta}^{\prime}$ can be rewritten as

$$
\mathcal{I}_{\delta}^{\prime}=\mathrm{e}^{i \lambda^{\frac{1}{2}} Q_{0}(\rho)} \int_{-\infty}^{\infty} g(u) \mathrm{e}^{i \lambda^{\frac{1}{2}}\left(\frac{u^{3}}{3}-\alpha u\right)} d u,
$$

where, recalling that $\sigma=\sigma(u)$,

$$
\begin{aligned}
g(u) & =\frac{t^{\frac{3}{2}}}{c^{2}\left(\sigma^{2}+1\right)} h\left(\frac{\delta}{t} \sigma-\frac{d}{c}\right)\left(\frac{d u}{d \sigma}\right)^{-1}\left(\frac{d \sigma}{d x}\right)^{-1} \\
& =\frac{\delta t^{\frac{1}{2}}}{c^{2}\left(\sigma^{2}+1\right)} h\left(\frac{\delta}{t} \sigma-\frac{d}{c}\right)\left(\frac{d u}{d \sigma}\right)^{-1} .
\end{aligned}
$$

Thus by Proposition 2.2 we obtain the following asymptotic expansion in $\lambda$ for $\mathcal{I}_{\delta}$. We will write $\|u\|_{H^{N}}$ for the Sobolev $N$-norm of a (smooth) function $u$ (the sum of the $L_{1}$-norms of the first $N$ derivatives).

Proposition 4.3. Let $g \in C_{c}^{\infty}, t=2 \pi n \lambda^{-\frac{1}{2}}, \gamma=\left(\begin{array}{ll}a & b \\ c & d\end{array}\right) \in \Gamma, c>0, N \geq 0$.

If $\operatorname{Support}(g)$ does not contain a non-empty neighborhood of the origin, then

$$
\mathcal{I}_{\delta}^{\prime}=O\left(\lambda^{-(N+2) / 6}|| h \|_{H^{N+3}}\right) .
$$

Otherwise

$$
\begin{aligned}
& \mathcal{I}_{\delta}^{\prime}=\mathrm{e}^{i \lambda^{\frac{1}{2}}} Q_{0}(\rho) 2 \pi \lambda^{-1 / 6} \sum_{n=0}^{N} \frac{i^{n} A i^{(n)}(0)}{\lambda^{n / 6} n !} \sum_{j=0}^{n}\left(\begin{array}{c}
n \\
j
\end{array}\right)\left(i \alpha \lambda^{1 / 2}\right)^{n-j} g^{(j)}(0) \\
& +O\left(\lambda^{-(N+2) / 6}|| h \|_{H^{N+3}}\right) .
\end{aligned}
$$

Moreover, the terms $g^{(j)}(0)$ are bounded; in fact, $g(u)$ is smooth. In particular, for $g$ given by (4.20)

$$
\begin{aligned}
g(0)= & \frac{\delta t^{\frac{1}{2}}}{c^{2}\left(1+\widetilde{\sigma}_{0}^{2}\right)} h\left(\frac{\delta}{t} \widetilde{\sigma}_{0}-\frac{d}{c}\right)\left(\frac{d u}{d \sigma}\right)^{-1} \\
g^{(1)}(0)=t^{-\frac{1}{2}} & \frac{h^{\prime}\left(\frac{\delta}{t} \widetilde{\sigma}_{0}-\frac{d}{c}\right)\left(\frac{d u}{d \sigma}\right)^{-2}}{c^{2}\left(1+\widetilde{\sigma}_{0}^{2}\right)} \\
& -\delta t^{\frac{1}{2}} \frac{h\left(\frac{\delta}{t} \widetilde{\sigma}_{0}-\frac{d}{c}\right)\left(2 \widetilde{\sigma}_{0}\left(\frac{d u}{d \sigma}\right)^{-2}+\left(\frac{d^{2} u}{d \sigma^{2}}\right)^{-1}\left(1+\widetilde{\sigma}_{0}^{2}\right)\right)}{c^{2}\left(1+\widetilde{\sigma}_{0}^{2}\right)^{2}},
\end{aligned}
$$

where $\widetilde{\sigma}_{0}=1+O\left(\lambda^{-1}\right)$. 
Proof. The proof of this statement will follow directly by applying Proposition 2.2 (with $\nu=\lambda^{1 / 2}$ ) to the integral (4.19), after showing that $g(u)$ is a smooth function with compact support.

Assume first that Support $(g)$ contains a neighborhood of the origin. Then for sufficiently large $\lambda, \rho$ is arbitrarily close to $\rho_{0}=1$ and $\sqrt{\alpha}$ is small. Thus the relationship $u=u(\sigma(x))$ given by the transformation (4.10) is analytic, and $[-\sqrt{\alpha}, \sqrt{\alpha}] \subset \operatorname{Support}(g)$. Furthermore $h \in C_{c}^{\infty}$, and $\sigma \in \mathcal{D}$ implies that $\left(\sigma^{2}+1\right)$ $\neq 0$. Thus it follows by (4.20) that $g$ is smooth and that $\operatorname{Support}(g)=\operatorname{Support}(h)$.

If on the other hand $\operatorname{Support}(g)$ does not contain a non-empty neighborhood of the origin, then, given $\epsilon>0$ small and for large enough $\lambda$,

$$
[-\sqrt{\alpha}-\epsilon, \sqrt{\alpha}+\epsilon] \cap \operatorname{Support}(g)=\emptyset .
$$

Thus the only contribution to the integral is from the error term. The error term can be simplified. First of all,

$$
\begin{array}{r}
\sum_{j=0}^{N+1}\left(\begin{array}{c}
N+1 \\
j
\end{array}\right)\left|\alpha \lambda^{1 / 2}\right|^{N+1-j}\left(\|g\|_{L_{1}}+\left\|g^{(j+2)}\right\|_{L_{1}}\right) \\
\leq 2\|g\|_{H^{N+3}}\left(\left|\alpha \lambda^{1 / 2}\right|+1\right)^{N+1} .
\end{array}
$$

Finally, since $\alpha=O\left(\lambda^{-1}\right)$ (see (4.18)), it follows that for large $\lambda$ the error term is as claimed.

Since we are interested in evaluating $g^{(n)}(0)$, we need to find that $\widetilde{\sigma}_{0} \leftrightarrow u=0$. So from (4.10), (4.15), (4.16), and (4.8) we have that $\widetilde{\sigma}_{0}$ is the solution to

$$
F(\sigma)=\sigma+\rho^{-1} \log \left(\frac{2 \mathrm{e}^{-1}}{\rho^{2}\left(\sigma^{2}+1\right)}\right)=0 .
$$

Note that $F$ is continuous $\forall \sigma \in \mathbb{R}$ and $\rho \neq 0$. When $\rho=1, F(1)=0$; thus $\widetilde{\sigma}_{0}=1$. When $\rho>1, F(2)>0$, and $F(0)<0$, so by the intermediate value theorem there exists $\widetilde{\sigma}_{0} \in(0,2)$ such that $F\left(\widetilde{\sigma}_{0}\right)=0$. Finally, since $\rho=\sqrt{\frac{\lambda}{\lambda-\frac{1}{4}}}$, from (4.23) we see that for $\lambda$ large,

$$
\widetilde{\sigma}_{0}=1+O\left(\lambda^{-1}\right)
$$

The proof is complete.

4.2. Higher order coefficients. The higher order Fourier coefficients of $\varphi_{i r}$ have the same form as the those of a cusp form. Wolpert has already studied the asymptotics of these Wol99; hence in this section we quickly review Wolpert's results and adapt them to our situation.

By (1.9) and (1.7) we have that

$$
\mathcal{I}_{m}^{\prime}=a_{m} \int_{t y=1}(\operatorname{Im}(\gamma z))^{\frac{1}{2}} K_{i r}(2 \pi|m| \operatorname{Im}(\gamma z)) \mathrm{e}^{i 2 \pi \operatorname{Re}(m \gamma z+n z)} h(x) d x .
$$

Now in view of Definition 3.1 of [Wol99] we similarly define

Definition 4.4. For $\lambda=\frac{1}{4}+r^{2}>\frac{1}{4}, m$ a non-zero integer and $z=x+i y, y>0$, let $s=2 \pi m \lambda^{-\frac{1}{2}}$ and $\mathcal{K}_{s}(z)=\mathrm{e}^{\pi \lambda^{\frac{1}{2}} / 2} \sqrt{2 \pi|m| y} K_{i r}(2 \pi|m| y) \mathrm{e}^{i 2 \pi m x}$ for the $K$-Bessel function $K_{i r}$. 
With this definition

$$
\mathcal{I}_{m}^{\prime}=(2 \pi|m|)^{-\frac{1}{2}} a_{m} \mathrm{e}^{-\pi \lambda^{\frac{1}{2}} / 2} \int_{t y=1} \mathcal{K}_{s}(\operatorname{Im}(\gamma z)) \mathrm{e}^{i 2 \pi \operatorname{Re}(n z)} h(x) d x .
$$

Remark 4.5. In the following propositions the notation is as in Wol99, with the following exceptions:

- We use $\widehat{\beta}$ and $\widehat{Q_{0}}$ instead of Wolpert's $\alpha$ and $Q_{0}$. This is in order to distinguish them from our previously used expressions. For further definitions of the terms involved, see Wol99.

- Also $\varphi_{i r}^{Y}$ is to be a truncated form of $\varphi_{i r}$; see Lemma A.1 which is the analog of Lemma 6.1 from Wol99. Because of this, the proofs in WWol99] should be modified to use Lemma A.1 instead of Lemma 6.1 of [Wol99].

From Theorem 4.8 of [Wol99] we have

Proposition 4.6 (Wolpert). Given $0<t_{0}<t_{1}$, choose $\beta>4$ and define $\widehat{\beta}$ by $(\widehat{\beta}-1)^{1 / 2}=2 t_{1}+(\beta-1)^{1 / 2}$. Let $s=2 \pi m \lambda^{-1 / 2}, t=2 \pi n \lambda^{-1 / 2}$, and, given $\gamma=\left(\begin{array}{ll}a & b \\ c & d\end{array}\right) \in \Gamma, c>0$, let $\tau=c^{2}|s t|^{-1}$.

For $t_{0} \leq t \leq t_{1}, \lambda$ large, and $N \geq 0$, then,

$$
\begin{aligned}
& \sum_{m \neq 0} \mathcal{I}_{m}^{\prime}= 2^{1 / 2} 3^{-1 / 3} \pi^{3 / 2} \lambda^{-5 / 12} \\
& \times \sum_{m<0} a_{m} \mathrm{e}^{-\pi \lambda^{1 / 2} / 2}|s|^{-1 / 2} \mathrm{e}^{i \lambda^{1 / 2} \widehat{Q_{0}}-i \frac{\pi}{4}} \sum_{k=0}^{N} \frac{i^{k} A i^{(k)}(0) G_{k}(\tau, t)}{\left(3 \lambda^{1 / 2}\right)^{\frac{k}{3}} k !} \\
&+O\left(\lambda^{-(2 N+1) / 12}\left\|\varphi_{i r}^{Y}\right\|_{L_{2}}\|h\|_{H^{N+3}}\right),
\end{aligned}
$$

where the coefficients $G_{k}(\tau, t)$ are supported in the interval $\beta<\tau^{-1}+2<\widehat{\beta}, \varphi_{i r}^{Y}$ is as in Lemma A.1, and the remainder constant depends on $t_{0}, t_{1}, \beta$, and $\gamma$.

Sketch of proof. The proof of this statement is long and can be found in detail in Wol99. Here we just give a sketch of the proof.

First use a partition $\left\{g_{j}\right\}_{j=0}^{3}$ of unity for $[0, \infty)$ to obtain $\sum_{m \neq 0} \mathcal{I}_{m}^{\prime}=\sum_{j=0}^{3} J_{j}(t, s)$. A careful analysis of the integrals $J_{j}(t, s)$ yields

$$
\begin{aligned}
& \left|J_{0}(t, s)\right|=O\left(\mathrm{e}^{-c^{\prime} \lambda^{1 / 2}}\left\|\varphi_{i r}^{Y}\right\|_{L_{2}}\right), \\
& \left|J_{1}(t, s)\right|=O\left(\lambda^{-3 / 4}\left\|\varphi_{i r}^{Y}\right\|_{L_{2}}\right), \\
& \left|J_{3}(t, s)\right|=O\left(\lambda^{-3 / 4}|| \varphi_{i r}^{Y} \|_{L_{2}}\right),
\end{aligned}
$$

for $0<c^{\prime}<\infty$. The case of $J_{2}(t, s)$ provides us with the actual sum as follows: An analysis of the phase functions of the integrals corresponding to $J_{2}(t, s)$ tells us that the phase functions can be written as a cubic monomial (see Proposition 3.3 of [Wol99]). Using the analog of Proposition 2.2 with $\alpha=0$ (Proposition 2.6 of Wol99) yields the required asymptotic expansion for each integral. For the error bound to include the contributions of the sum on $m<0$, an application of a bound on $\sum\left|a_{m}\right|^{2}$ is required, so again use Lemma A.1 
4.3. Recall that from (1.9) and (4.1)

$$
\mathcal{J}^{\prime}=a_{0_{+}} \mathcal{I}_{+}^{\prime}+a_{0_{-}} \mathcal{I}_{-}^{\prime}+\sum_{m \neq 0} \mathcal{I}_{m}^{\prime}
$$

Thus, by Proposition 4.6 and Proposition 4.3 we obtain

Corollary 4.7. Given $0<t_{0}<t_{1}$, choose $\beta>4$ and define $\hat{\beta}$ by $(\hat{\beta}-1)^{1 / 2}=$ $2 t_{1}+(\beta-1)^{1 / 2}$. Let $s=2 \pi m \lambda^{-1 / 2}, t=2 \pi n \lambda^{-1 / 2}$, and, given $\gamma=\left(\begin{array}{ll}a & b \\ c & d\end{array}\right) \in \Gamma, c>0$, let $\tau=c^{2}|s t|^{-1}$.

For $t_{0} \leq t \leq t_{1}, \lambda$ large, and $N \geq 0$, then,

$$
\begin{gathered}
\mathcal{J}^{\prime}=2 \pi \lambda^{-1 / 6} \mathcal{A}(c, d) \mathrm{e}^{i \lambda^{\frac{1}{2}}} Q_{0}(\rho) \\
\times \sum_{n=0}^{N-1} \frac{i^{n} A i^{(n)}(0)}{\lambda^{n / 6} n !} \sum_{j=0}^{n}\left(\begin{array}{c}
n \\
j
\end{array}\right)\left(i \alpha \lambda^{1 / 2}\right)^{n-j} g^{(j)}(0) \\
+2^{1 / 2} 3^{-1 / 3} \pi^{3 / 2} \lambda^{-5 / 12} \sum_{m<0} a_{m} \mathrm{e}^{-\pi \lambda^{1 / 2} / 2}|s|^{-1 / 2} \mathrm{e}^{i \lambda^{1 / 2} \widehat{Q_{0}}-i \frac{\pi}{4}} \\
\times \sum_{k=0}^{N} \frac{i^{k} A i^{(k)}(0) G_{k}(\tau, t)}{\left(3 \lambda^{1 / 2}\right)^{k / 3} k !} \\
+O\left(\lambda^{-(2 N+1) / 12}\left\|\varphi_{i r}^{Y}\right\|_{L_{2}}\|h\|_{H^{N+3}}\right),
\end{gathered}
$$

where the coefficients $G_{k}(\tau, t)$ are supported in the interval $\beta<\tau^{-1}+2<\hat{\beta}, \varphi_{i r}^{Y}$ is as in Lemma A.1, the remainder constant depends on $t_{0}, t_{1}, \beta$, and $\gamma$, and the function $\mathcal{A}(c, d)$ is given by

$$
\mathcal{A}(c, d)= \begin{cases}a_{0_{+}}, & \text {whenever } \\ a_{0_{-}}, & \text {whenever }\left(-\frac{c \widetilde{\sigma}_{0}}{c h_{0}+d}, \frac{c \widetilde{\sigma}_{0}}{c h_{1}+d}\right) \cap\left[t_{0}, t_{1}\right] \neq \emptyset \\ 0, & \text { otherwise, }\end{cases}
$$

where $\operatorname{Support}(h) \subset\left(h_{0}, h_{1}\right)$.

Proof. Since Support $(h) \subset\left(h_{0}, h_{1}\right)$, by (4.20) we have

$$
h_{0}<\frac{\delta}{t} \widetilde{\sigma}_{0}-\frac{d}{c}<h_{1},
$$

where $\widetilde{\sigma}_{0} \in(0,2)$ (recall that by (4.24) $\widetilde{\sigma}_{0}=1+O\left(\lambda^{-1}\right)$ as $\lambda \rightarrow \infty$ ). Thus

$$
\frac{c \widetilde{\sigma}_{0}}{c h_{1}+d}<\delta t<\frac{c \widetilde{\sigma}_{0}}{c h_{0}+d} .
$$

So $\mathcal{I}_{+}^{\prime}$ only contributes (other than with a remainder term) when

$$
\left(\frac{c \widetilde{\sigma}_{0}}{c h_{1}+d}, \frac{c \widetilde{\sigma}_{0}}{c h_{0}+d}\right) \cap\left[t_{0}, t_{1}\right] \neq \emptyset .
$$

Similarly $\mathcal{I}_{-}^{\prime}$ contributes when

$$
\left(-\frac{c \widetilde{\sigma}_{0}}{c h_{0}+d},-\frac{c \widetilde{\sigma}_{0}}{c h_{1}+d}\right) \cap\left[t_{0}, t_{1}\right] \neq \emptyset .
$$

This finishes the proof. 
Remark 4.8. Notice that the support of $g$ (hence $h$ ) restricts the range where there is a contribution from the zeroth order coefficient. Later on we shall be interested in controlling when we have a contribution arising from $a_{0}(y)$.

Remark 4.9. In the definition of the phase function $\psi_{\delta}$ (see (4.3)) it appears that the factor $\lambda^{\frac{1}{2}}$ is artificial; it would seem more natural to use $r$. The choice was made for the following reason: if one uses $r$, then the phase function $\psi_{\delta}$ can be written (after a change of variables) as a cubic monomial (which makes the calculation of 4.1 easier), while Wolpert's phase function instead becomes a cubic with two coalescing stationary points (and hence has to be treated with Proposition 2.2. This makes the calculations done in $\$ 4.2$ much more involved.

\section{The COEFFicient RELATION}

In this section we put together all the pieces developed in the previous sections and obtain a relationship between the Fourier coefficients.

In Corollary 3.4 we have an asymptotic evaluation of $\mathcal{J}$, and in Corollary 4.7 we have found another evaluation of the same integral. Hence, equating them and simplifying, we obtain

Theorem 5.1 (Coefficient Relation). Given $0<t_{0}<t_{1}$, choose $\beta>4$ and define $\hat{\beta}$ by $(\hat{\beta}-1)^{1 / 2}=2 t_{1}+(\beta-1)^{1 / 2}$. Let $s=2 \pi m \lambda^{-1 / 2}, t=2 \pi n \lambda^{-1 / 2}$, and, given $\gamma=\left(\begin{array}{ll}a & b \\ c & d\end{array}\right) \in \Gamma, c>0$, let $\tau=c^{2}|s t|^{-1}$.

For $t_{0} \leq t \leq t_{1}, \lambda$ large, and $1 \leq N \leq 4$, then,

$$
\begin{aligned}
a_{n} \mathrm{e}^{-\pi \lambda^{\frac{1}{2}} / 2}=\frac{2^{\frac{2}{3}} t^{\frac{1}{2}} \mathcal{A}(c, d) \mathrm{e}^{-i \lambda^{\frac{1}{2}}} Q_{0}(\rho)}{A i(0)+\frac{2^{\frac{1}{3}}}{70 \lambda^{\frac{2}{3}}} A i^{\prime}(0)} \\
\quad \times \sum_{n=0}^{N-1} \frac{A i^{(n)}(0)}{i^{n} \lambda^{n / 6} n !} \sum_{j=0}^{n}\left(\begin{array}{c}
n \\
j
\end{array}\right)\left(-i \alpha \lambda^{1 / 2}\right)^{n-j} g^{(j)}(0) \\
+\frac{2^{\frac{1}{6}} 3^{-\frac{1}{3}} \pi^{\frac{1}{2}} \lambda^{-\frac{1}{4}}}{A i(0)+\frac{2^{\frac{1}{3}}}{70 \lambda^{\frac{2}{3}}} A i^{\prime}(0)} \sum_{m>0} a_{m} \mathrm{e}^{-\pi \lambda^{\frac{1}{2}} / 2} s^{-\frac{1}{2}} t^{\frac{1}{2}} \mathrm{e}^{-i \lambda^{\frac{1}{2}} \widehat{Q_{0}}+i \frac{\pi}{4}} \\
\times \sum_{k=0}^{N} \frac{A i^{(k)}(0)}{i^{k}\left(3 \lambda^{\frac{1}{2}}\right)^{\frac{k}{3}} k !}+O\left(\lambda^{-(2 N-1) / 12}\left\|\varphi_{i r}^{Y}\right\|_{L_{2}}\|h\|_{H^{N+3}}\right),
\end{aligned}
$$

where the coefficients $G_{k}(\tau, t)$ are supported in the interval $\beta<\tau^{-1}+2<\hat{\beta}$, the remainder constant depends on $t_{0}, t_{1}, \beta$, and $\gamma, \varphi_{i r}^{Y}$ is as in Lemma A.1, and $\mathcal{A}(c, d)$ is given by

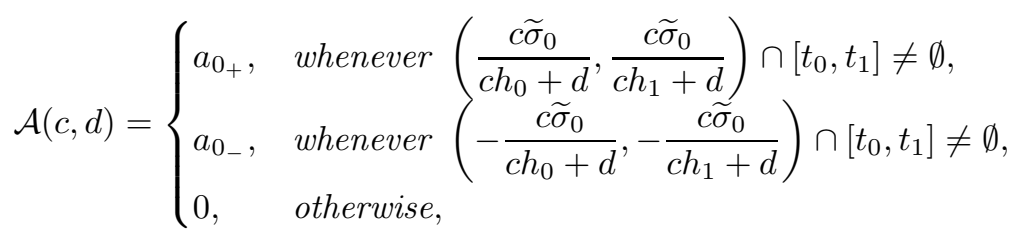

where $\operatorname{Support}(h) \subset\left(h_{0}, h_{1}\right)$.

When $N=1$ we obtain a much simpler expression for the coefficients, since in this case we need only consider the "leading term" in the asymptotic expansion from Corollary 3.4 . 
Corollary 5.2. Situation as above with $N=1$; then

$$
\begin{aligned}
a_{n} \mathrm{e}^{-\pi \lambda^{\frac{1}{2}} / 2}= & 2^{\frac{2}{3}} t^{\frac{1}{2}} \mathcal{A}(c, d) \mathrm{e}^{-i \lambda^{\frac{1}{2}} Q_{0}(\rho)} g(0) \\
& +2^{\frac{1}{6}} 3^{-\frac{1}{3}} \pi^{\frac{1}{2}} \lambda^{-\frac{1}{4}} \sum_{m>0} a_{m} \mathrm{e}^{-\pi \lambda^{\frac{1}{2}} / 2} s^{-\frac{1}{2}} t^{\frac{1}{2}} \mathrm{e}^{-i \lambda^{\frac{1}{2}} \widehat{Q_{0}}+i \frac{\pi}{4}} \\
& \times\left(\overline{G_{0}(\tau, t)}-i \frac{\Gamma\left(\frac{2}{3}\right)}{\Gamma\left(\frac{1}{3}\right)} \overline{\frac{G_{1}(\tau, t)}{\lambda^{1 / 6}}}\right)+O\left(\lambda^{-1 / 12}\left\|\varphi_{i r}^{Y}\right\|_{L_{2}}\|h\|_{H^{4}}\right) .
\end{aligned}
$$

Proof. This follows directly from Theorem 5.1 by considering only the leading term in the asymptotic expansion given in Corollary 3.4, together with the fact that

$$
\frac{A i^{\prime}(0)}{A i(0)}=3^{1 / 3} \frac{\Gamma\left(\frac{2}{3}\right)}{\Gamma\left(\frac{1}{3}\right)} \text {. }
$$

Notice that the zeroth Fourier coefficient $a_{0}(y)$ does not contribute if and only if

$$
\begin{gathered}
\left(\frac{c \widetilde{\sigma}_{0}}{c h_{0}+d}, \frac{c \widetilde{\sigma}_{0}}{c h_{1}+d}\right) \cap\left[t_{0}, t_{1}\right]=\emptyset, \\
\text { or } \\
\left(-\frac{c \widetilde{\sigma}_{0}}{c h_{0}+d},-\frac{c \widetilde{\sigma}_{0}}{c h_{1}+d}\right) \cap\left[t_{0}, t_{1}\right]=\emptyset,
\end{gathered}
$$

where $\operatorname{Support}(h) \subset\left[h_{0}, h_{1}\right]$, and $\widetilde{\sigma}_{0}=1+O\left(\lambda^{-1}\right)$. Hence given $t_{0}$ and $t_{1}$ it is always possible to choose $\gamma=\left(\begin{array}{ll}a & b \\ c & d\end{array}\right) \in \Gamma$ and a test function $h$ (say with a small enough support) such that (5.2) is satisfied. Thus an application of Lemma A.1 as in Corollary 4.9 of [Wol99] gives

Corollary 5.3. For $t_{0} \leq t \leq t_{1}, t_{0}, t_{1}$ satisfying (5.2),

$$
a_{n}=O\left(\lambda^{1 / 4}\left\|\varphi_{i r}^{Y}\right\|_{L_{2}}\|h\|_{H^{7}} \mathrm{e}^{\pi \lambda^{1 / 2} / 2}\right),
$$

where the remainder constants depend on $\gamma, \beta, t_{0}$ and $t_{1}$.

\section{Applications}

In this section we use the coefficient relation, in particular Corollary 5.2, to obtain asymptotic relations among the classical divisor functions and among Kloosterman sums.

6.1. Asymptotic relations of Kloosterman sums. Let $\Gamma \subset S L_{2}(\mathbb{R})$ be a finitely generated non-cocompact Fuchsian group of the first kind; it has a finite number of inequivalent cusps $\{\alpha\}$. Let $\Gamma_{\alpha}$ be the stability group of $\alpha$; it is a cyclic infinite group generated by $\gamma_{\alpha}$, say. We may assume that $\infty$ is one of the cusps by, if necessary, conjugating $\Gamma$, in which case we have $\Gamma_{\infty}=\left\{\left(\begin{array}{cc}1 & m \\ 0 & 1\end{array}\right): m \in \mathbb{Z}\right\}$. Define the Eisenstein series

$$
\frac{1}{2} E_{\alpha}(z, s, \chi)=\sum_{\gamma \in \Gamma_{\alpha} \backslash \Gamma} \chi(\gamma) \operatorname{Im}\left(\sigma_{\alpha}^{-1} \gamma z\right)^{s}, \quad z \in \mathbb{H},
$$

where $\sigma_{\alpha} \in S L_{2}(\mathbb{R})$ satisfies $\sigma_{\alpha} \infty=\alpha$ and $\sigma_{\alpha}^{-1} \Gamma_{\alpha} \sigma_{\alpha}=\Gamma_{\infty}$. The character $\chi$ satisfies $\chi\left(\sigma_{\alpha}\left(\begin{array}{ll}1 & 1 \\ 0 & 1\end{array}\right) \sigma_{\alpha}^{-1}\right)=1$ for each $\alpha$.

This Eisenstein series is a-priori defined only for $\operatorname{Re}(s)>1$, but has meromorphic continuation over the complex $s$-plane. In the half plane $\operatorname{Re}(s) \geq 1 / 2$ the 
Eisenstein series is holomorphic except for a simple pole at $s=1$. Moreover, it is $\Gamma$-automorphic,

$$
E_{\alpha}(\gamma z, s, \chi)=\overline{\chi(\gamma)} E_{\alpha}(z, s, \chi)
$$

so we can apply the results of $\$ 5$ to obtain asymptotic relations among its Fourier coefficients.

To describe the Fourier coefficients, consider the Kloosterman sum

$$
S_{\alpha \beta}(m, n, \chi, f ; c)=\sum_{\gamma \in \Gamma_{\infty} \backslash \sigma_{\alpha}^{-1} \Gamma \sigma_{\beta} / \Gamma_{\infty}}^{\prime} \chi\left(\sigma_{\alpha} \gamma \sigma_{\beta}^{-1}\right) \mathrm{e}^{i 2 \pi\left(n \frac{a}{c}+m \frac{d}{c}\right)},
$$

where as usual $\gamma=\left(\begin{array}{ll}a & b \\ c & d\end{array}\right)$, and the prime on the sum indicates that it is to be taken only for those $\gamma$ 's that have their lower left entry $c$ fixed. Note that this Kloosterman sum is defined for $c \in C_{\alpha \beta}=\left\{c>0:\left(\begin{array}{c}* * \\ c *\end{array}\right) \in \sigma_{\alpha}^{-1} \Gamma \sigma_{\beta}\right\}$.

The Fourier expansion of $\frac{1}{2} E_{\alpha}(z, s, \chi)$ is then

$$
\begin{aligned}
\frac{1}{2} E_{\alpha}(z, s, \chi)=\delta_{\alpha \beta} y^{s}+ & \phi_{\alpha \beta}(s, \chi) y^{1-s} \\
& +2 \sum_{n \neq 0} \phi_{\alpha \beta}(n, s, \chi)|n|^{\frac{1}{2}} y^{\frac{1}{2}} K_{s-\frac{1}{2}}(2 \pi|n| y) \mathrm{e}^{i 2 \pi n x}
\end{aligned}
$$

where

$$
\phi_{\alpha \beta}(s, \chi)=\sqrt{\pi} \frac{\Gamma\left(s-\frac{1}{2}\right)}{\Gamma(s)} \sum_{c \in C_{\alpha \beta}} \frac{S_{\alpha \beta}(0,0, \chi, f ; c)}{c^{2 s}}
$$

and

$$
\phi_{\alpha \beta}(n, s, \chi)=|n|^{s-1} \frac{\pi^{s}}{\Gamma(s)} \sum_{c \in C_{\alpha \beta}} \frac{S_{\alpha \beta}(n, 0, \chi, f ; c)}{c^{2 s}} .
$$

For simplicity we assume that $\Gamma$ has a unique cusp, and by conjugation we might as well say it is at $\infty$. Furthermore we let $s=\frac{1}{2}+i r$ and $\chi=1$, so

$$
\begin{aligned}
\frac{1}{2} E_{\infty}\left(z, \frac{1}{2}+i r, 1\right)=y^{\frac{1}{2}+i r} & +\phi_{\infty}\left(\frac{1}{2}+i r, 1\right) y^{\frac{1}{2}-i r} \\
& +2 \sum_{n \neq 0} \phi_{\infty}\left(n, \frac{1}{2}+i r, 1\right)|n|^{\frac{1}{2}} y^{\frac{1}{2}} K_{i r}(2 \pi|n| y) \mathrm{e}^{i 2 \pi n x}
\end{aligned}
$$

where $\phi_{\infty}$ is $\phi_{\alpha \beta}$ with $\alpha=\beta=\infty$. Hence, as is seen from the definitions (6.5) and (6.6), the Fourier coefficients are sums of Kloosterman sums.

Theorem 6.1 (Relations among sums of Kloosterman sums). Let $\Gamma \subset S L_{2}(\mathbb{R})$ be a finitely generated non-cocompact Fuchsian group of the first kind with a cusp at $\infty$. Given $0<t_{0}<t_{1}$, choose $\beta>4$ and define $\hat{\beta}$ by $(\hat{\beta}-1)^{1 / 2}=2 t_{1}+(\beta-1)^{1 / 2}$. Let $s=2 \pi m \lambda^{-1 / 2}, t=2 \pi n \lambda^{-1 / 2}$, and, given $\gamma=\left(\begin{array}{cc}a & b \\ c & d\end{array}\right) \in \Gamma, c>0$, let $\tau=c^{2}|s t|^{-1}$. 
For $t_{0} \leq t \leq t_{1}, \lambda=\frac{1}{4}+r^{2}$ large, then,

$$
\begin{aligned}
& n^{i r-\frac{1}{2}} \sum_{\tilde{c} \in C_{\infty}} \frac{S_{\infty}(n, 0,1, f ; \tilde{c})}{\tilde{c}^{1+2 i r}} \mathrm{e}^{-\pi \lambda^{\frac{1}{2}} / 2} \\
& =2^{\frac{1}{6}} \pi^{-i r} \lambda^{-\frac{1}{4}} n^{-\frac{1}{2}} \mathcal{A}^{\prime}(c, d) \mathrm{e}^{-i \lambda^{\frac{1}{2}} Q_{0}(\rho)} g(0) \\
& +2^{\frac{1}{6}} 3^{-\frac{1}{3}} \pi^{\frac{1}{2}} \lambda^{-\frac{1}{4}} \sum_{m>0} m^{i r-\frac{1}{2}}\left(\sum_{\tilde{c} \in C_{\infty}} \frac{S_{\infty}(m, 0,1, f ; \tilde{c})}{\tilde{c}^{1+2 i r}}\right) \mathrm{e}^{-\pi \lambda^{\frac{1}{2}} / 2} \mathrm{e}^{-i \lambda^{\frac{1}{2}} \widehat{Q_{0}}+i \frac{\pi}{4}} \\
& \quad \times\left(\overline{G_{0}(\tau, t)}-i \frac{\Gamma\left(\frac{2}{3}\right)}{\Gamma\left(\frac{1}{3}\right)} \frac{\overline{G_{1}(\tau, t)}}{\lambda^{1 / 6}}\right)+O\left(\lambda^{-1 / 12}\left\|E_{\infty}(z, s, 1)^{Y}\right\|_{L_{2}}\|h\|_{H^{4}}\right),
\end{aligned}
$$

where the coefficients $G_{k}(\tau, t)$ are supported in the interval $\beta<\tau^{-1}+2<\hat{\beta}$, $\left\|E_{\infty}(z, s, 1)^{Y}\right\|_{L_{2}}$ is as in Lemma A.2, the remainder constant depends on $t_{0}, t_{1}, \beta$, and $\gamma$, and the function $\mathcal{A}^{\prime}(c, d)$ is given by

$$
\mathcal{A}^{\prime}(c, d)= \begin{cases}\Gamma\left(\frac{1}{2}+i r\right), & \text { whenever }\left(\frac{c \widetilde{\sigma}_{0}}{c h_{0}+d}, \frac{c \widetilde{\sigma}_{0}}{c h_{1}+d}\right) \cap\left[t_{0}, t_{1}\right] \neq \emptyset, \\ \sqrt{\pi} \Gamma(i r) \sum_{\tilde{c} \in C_{\infty}} \frac{S_{\infty}(n, 0,1, f ; \tilde{c})}{\tilde{c}^{1+2 i r}}, & \text { whenever }\left(-\frac{c \widetilde{\sigma}_{0}}{c h_{0}+d},-\frac{c \widetilde{\sigma}_{0}}{c h_{1}+d}\right) \cap\left[t_{0}, t_{1}\right] \neq \emptyset, \\ 0, & \text { otherwise, }\end{cases}
$$

where $\operatorname{Support}(h) \subset\left(h_{0}, h_{1}\right)$.

Proof. Apply Corollary 5.2 with $a_{n}=2|n|^{\frac{1}{2}} \phi_{\infty}\left(n, \frac{1}{2}+i r, 1\right), a_{0+}=1$, and $a_{0-}=$ $\phi_{\infty}\left(\frac{1}{2}+i r\right)$.

6.2. Asymptotic relations among divisor functions. The coefficient relation for the divisor functions will follow directly from Corollary 5.2 when we restrict ourselves to considering the real analytic Eisenstein series

$$
\varphi_{i r}(z)=\frac{1}{2} \sum_{\substack{c, d \in \mathbb{Z} \\(c, d)=1}} y^{s}|c z+d|^{-2 s},
$$

where we recall that $s=\frac{1}{2}+i r$. The Fourier development of $\varphi_{i r}$ can be given explicitly (see for instance [Bru94], pp. 9, and [Iwa97], pp. 236, 237):

$$
\begin{aligned}
\varphi_{i r}(z) & =y^{\frac{1}{2}+i r}+\frac{\pi^{\frac{1}{2}} \Gamma(i r) \zeta(2 i r)}{\Gamma\left(\frac{1}{2}+i r\right) \zeta(2 i r+1)} y^{\frac{1}{2}-i r} \\
& +\frac{\pi^{\frac{1}{2}+i r}}{\Gamma\left(\frac{1}{2}+i r\right) \zeta(2 i r+1)} \sum_{m \neq 0} \frac{\mathrm{d}_{2 i r}(|m|)}{|m|^{\frac{1}{2}+i r}} 2(|m| y)^{\frac{1}{2}} K_{i r}(2 \pi|m| y) \mathrm{e}^{i 2 \pi m x}
\end{aligned}
$$

where

$$
\mathrm{d}_{q}(m)=\sum_{\substack{d \mid m \\ d>0}} d^{q}
$$


is the divisor function, and $\zeta(\omega)$ is the analytic continuation of the Riemann zeta function.

By (6.9) the Fourier coefficients of $\varphi_{i r}$ are given by

$$
\begin{aligned}
& a_{0_{+}}=1, \\
& a_{0_{-}}=\frac{\pi^{\frac{1}{2}} \Gamma(i r) \zeta(2 i r)}{\Gamma\left(\frac{1}{2}+i r\right) \zeta(2 i r+1)},
\end{aligned}
$$

and for $m \neq 0$

$$
a_{m}=\frac{2 \pi^{\frac{1}{2}+i r}}{\Gamma\left(\frac{1}{2}+i r\right) \zeta(2 i r+1)} \frac{\mathrm{d}_{2 i r}(|m|)}{|m|^{i r}} .
$$

Corollary 6.2 (Relations among divisor functions). Given $0<t_{0}<t_{1}$, choose $\beta>4$ and define $\widehat{\beta}$ by $(\widehat{\beta}-1)^{1 / 2}=2 t_{1}+(\beta-1)^{1 / 2}$. Let $s=2 \pi m \lambda^{-1 / 2}, t=$ $2 \pi n \lambda^{-1 / 2}$, and, given $\gamma=\left(\begin{array}{ll}a & b \\ c & d\end{array}\right) \in S L_{2}(\mathbb{Z}), c>0$, let $\tau=c^{2}|s t|^{-1}$.

For $t_{0} \leq t \leq t_{1}, \lambda=\frac{1}{4}+r^{2}$ large, then,

$$
\begin{aligned}
& \frac{d_{2 i r}(n)}{n^{\frac{1}{2}+i r}}=2^{\frac{1}{6}} \pi^{-i r} \lambda^{-\frac{1}{4}} \mathcal{B}(c, d) \mathrm{e}^{-i \lambda^{\frac{1}{2}} Q_{0}(\rho)} g(0) \\
& +2^{\frac{1}{6}} 3^{-\frac{1}{3}} \pi^{\frac{1}{2}} \lambda^{-\frac{1}{4}} \sum_{m>0} \frac{d_{2 i r}(m)}{m^{\frac{1}{2}+i r}} \mathrm{e}^{-i \lambda^{\frac{1}{2}} \widehat{Q_{0}}+i \frac{\pi}{4}}\left(\begin{array}{c}
\left.\overline{G_{0}(\tau, t)}-i \frac{\Gamma\left(\frac{2}{3}\right)}{\Gamma\left(\frac{1}{3}\right)} \frac{\overline{G_{1}(\tau, t)}}{\lambda^{1 / 6}}\right) \\
+O\left(\lambda^{-1 / 12}\left\|\varphi_{i r}^{Y}\right\|_{L_{2}}\|h\|_{H^{4}}\right),
\end{array}\right.
\end{aligned}
$$

where $\mathcal{B}(c, d)$ is given by

$$
\mathcal{B}(c, d)= \begin{cases}\Gamma\left(\frac{1}{2}+i r\right) \zeta(2 i r+1), & \text { whenever }\left(\frac{c \widetilde{\sigma}_{0}}{d}, \frac{c \widetilde{\sigma}_{0}}{2 c+d}\right) \cap\left[t_{0}, t_{1}\right] \neq \emptyset \\ \Gamma(i r) \zeta(2 i r), & \text { whenever } \\ 0, & \text { otherwise, }\end{cases}
$$

the coefficients $G_{k}(\tau, t)$ are supported in the interval $\beta<\tau^{-1}+2<\widehat{\beta},\left\|\varphi_{i r}^{Y}\right\|_{L_{2}}$ is as in Lemma A.2, and the remainder constant depends on $t_{0}, t_{1}, \beta$, and $\gamma$.

Proof. The proof of this statement follows directly from applying Corollary 5.2 with (6.11) and (6.12).

Remark 6.3. The coefficient relation can also be obtained as a special case of Theorem 6.1] since the Eisenstein series given by (6.8) is the same one as the one obtained when one has $\Gamma=S L_{2}(\mathbb{Z})$ in (6.1).

\section{Appendix A.}

Here we present a slight modification (Lemma A.1 due to Wolpert) on Lemma 6.1 of Wol99. that provides an upper bound for the sum of the magnitude squared of the Fourier coefficients $a_{n}(y)$ of a real analytic Eisenstein series for $S L_{2}(\mathbb{Z})$.

Also we provide, using the Maass-Selberg relations, and some estimates of Hejhal Hej83 on the scattering matrix, an upper bound for the $L_{2}$-norm of $\varphi_{i r}^{Y}$ (see below for the definition). 
Let $\Gamma$ and $\varphi_{i r}$ be as before ( $\Gamma$ a finitely generated Fuchsian group of the first kind, and $\varphi_{i r}$ a $\Gamma$-automorphic eigenfunction of the hyperbolic Laplacian with eigenvalue $\lambda=\frac{1}{4}+r^{2}$ and Fourier development given by (1.1)).

A fundamental domain $\mathcal{F}$ is standard for a cusp $\alpha$ for $\Gamma$ provided that for $\gamma_{\alpha} \in \Gamma$ generating the stabilizer $\Gamma_{\alpha}$ there exists an $\sigma_{\alpha} \in S L_{2}(\mathbb{R})$ with $\sigma_{\alpha} \gamma_{\alpha} \sigma_{\alpha}^{-1}=\left(\begin{array}{cc}1 & \pm 1 \\ 0 & 1\end{array}\right)$ such that $\sigma_{\alpha} \mathcal{F} \subset\{0 \leq \operatorname{Re}(z) \leq 1\}$ and $\sigma_{\alpha} \mathcal{F} \cap\{\operatorname{Im}(z) \geq 1\}=\{0 \leq \operatorname{Re}(z)<$ $1, \operatorname{Im}(z) \geq 1\}$. Let $\mathcal{F}_{\alpha}=\sigma_{\alpha} \mathcal{F} \cap\{\operatorname{Im}(z) \geq 1\}$

For $z \in \mathcal{F}_{\alpha}$ define

$$
\varphi_{i r}^{Y}(z)= \begin{cases}\varphi_{i r}(z) & \text { for } \operatorname{Im}(z) \leq Y, \\ \varphi_{i r}(z)-a_{0}(y) & \text { for } \operatorname{Im}(z)>Y .\end{cases}
$$

Lemma A.1. The Fourier coefficients of $\varphi_{\text {ir }}$ for $\Gamma$ as above satisfy

$$
\sum_{n=1}^{N}\left|a_{n}(r)\right|^{2} \leq C\left\|\varphi_{i r}^{Y}\right\|_{L_{2}}^{2}(N+|r|) \sinh \pi|r|,
$$

for $|r|$ sufficiently large and for all positive integers $N$.

Proof. Since we are working with only one cusp $\alpha$ we might as well assume that it is $\alpha=\infty$, so that $\Gamma_{\alpha}=\Gamma_{\infty}$. Set $Y=4 \pi N|r|^{-1}$. Observe that since the $\Gamma$ images of $\{\operatorname{Im}(z) \geq Y\}$ are contained in $\left\{\operatorname{Im}(z) \leq Y^{-1}\right\}$, then $\varphi_{i r}^{Y}(z)=\varphi_{\text {ir }}(z)$ on $\left\{Y^{-1} \leq \operatorname{Im}(z) \leq Y\right\}$ and $\varphi_{i r}^{Y}(z)=\varphi_{i r}(z)-a_{0}(y)$ on $\{\operatorname{Im}(z)>Y\}$. In particular, on the entire horoball $\left\{\operatorname{Im}(z) \geq Y^{-1}\right\}$ the Fourier expansions relative to $\Gamma_{\infty}$ of $\varphi_{\text {ir }}$ and $\varphi_{i r}^{Y}$ agree except for the zeroth Fourier coefficient. Applying the arguments of Wol99] (Lemmas 6.1 and 6.2) completes the proof.

From the Maass-Selberg relation (see for instance $\S 12.8$ of [Bor97]) we find the $L_{2}$-norm of $\varphi_{i r}^{Y}$,

$$
\left\|\varphi_{i r}^{Y}\right\|_{L_{2}}^{2}=\int_{\mathcal{F}}\left|\varphi_{i r}^{Y}\right|^{2} d A=2 \log Y-\frac{\varphi^{\prime}\left(\frac{1}{2}+i r\right)}{\varphi\left(\frac{1}{2}+i r\right)}-r^{-1} \operatorname{Im}\left(\varphi\left(\frac{1}{2}+i r\right) Y^{-2 i r}\right),
$$

where $\varphi\left(\frac{1}{2}+i r\right)=a_{0_{-}}(r)$ is the scattering matrix (not to be confused with $\varphi_{i r}$, which is the Eisenstein series in consideration), and $Y=4 \pi N|r|^{-1}$ as in the proof of the above lemma (in what follows $Y$ is defined in the same way). The scattering matrix $\varphi(s)$ on the critical line $\operatorname{Re}(s)=\frac{1}{2}$ satisfies (Hej83 Theorem $11.8(\mathrm{C})$ )

$$
\left|\varphi\left(\frac{1}{2}+i r\right)\right|=1
$$

for $r \in \mathbb{R}$. Moreover, in the region given by $\frac{1}{2} \leq \operatorname{Re}(s) \leq \frac{3}{2}$ and $|\operatorname{Im}(s)| \geq 1,|\varphi(s)|$ is uniformly bounded: $|\varphi(s)| \leq(1+\sqrt{2}) Y^{2}$ (Proposition 12.4 of [Hej83]). Hence by combining this last inequality, A.3 and (A.2) together with Cauchy's inequalities we have the following bound for the norm.

Lemma A.2. Situation as above. Then

$$
\left\|\varphi_{i r}^{Y}\right\|_{L_{2}}^{2} \leq 2 \log Y+(1+\sqrt{2})\left(\frac{1}{R}+\frac{1}{r}\right) Y^{2},
$$

where $0<R<\operatorname{dist}\left(\frac{1}{2}+i r, \Omega^{c}\right)$, $\Omega$ being the domain of holomorphicity of the scattering matrix $\varphi(s)$. 
Combining this with Lemma A.1, we obtain

Lemma A.3. Situation as above. Then

$$
\sum_{n=1}^{N}\left|a_{n}(r)\right|^{2} \leq C \sinh \pi|r|(N+|r|)\left(\log \frac{N}{|r|}+\frac{N^{2}}{r^{2}}\left(1+O\left(r^{-1}\right)\right)\right),
$$

for $|r|$ sufficiently large and all positive integers $N$.

\section{REFERENCES}

[Bor97] Armand Borel, Automorphic forms on $S L_{2}(\mathbb{R})$, Cambridge University Press, 1997. MR 98j: 11028

[Bru94] R. W. Bruggeman, Families of Automorphic Forms, Birkhäuser-Verlag, 1994. MR 95k:11060

[CFU57] C. Chester, B. Friedman, and F. Ursell, An extension of the method of steepest descents, Proc. Cambridge Philos. Soc. 53 (1957), 599-611. MR 19:853a

[GS77] V. Guillemin and S. Sternberg, Geometric Asymptotics, Mathematical Surveys. Number 14, American Mathematical Society, 1977. MR 58:24404

[Hej83] D. A. Hejhal, The Selberg Trace Formula for $P S L_{2}(\mathbb{R})$ Volume 2, Lecture Notes in Mathematics 1001, Springer-Verlag, 1983. MR 86e:11040

[HW79] G. H. Hardy and E. M. Wright, An introduction to the theory of numbers, $5^{\text {th }}$ edition, Oxford University Press, 1979. MR 81i:10002

[Iwa97] H. Iwaniec, Topics in Classical Automorphic Forms, Graduate Studies in Mathematics, vol. 17, American Mathematical Society, 1997. MR 98e:11051

[Olv97] F. W. J. Olver, Asymptotics and Special Functions, A. K. Peters, 1997. MR 97i:41001

[Wol99] S. A. Wolpert, Asymptotic relations among Fourier coefficients of automorphic eigenfunctions, Preprint sent to publication, 1998.

[Zem65] A. H. Zemanian, Distribution Theory and Transform Analysis, McGraw-Hill Book Company, New York, 1965. MR 31:1556

Department of Mathematics, University of Maryland at College Park, College PARK, MARYLAND 20740

E-mail address: aap@math.umd.edu

Current address: P.O. Box 435294, San Ysidro, California 92173 